\title{
Treatment of thoracolumbar burst fractures: extended follow-up of a randomized clinical trial comparing orthosis versus no orthosis
}

\author{
*Jennifer C. Urquhart, PhD, ${ }^{1}$ Osama A. Alrehaili, MD, ${ }^{1}$ Charles G. Fisher, MD, MHSc, ${ }^{2}$ \\ Alyssa Fleming, ${ }^{1}$ Parham Rasoulinejad, MD, ${ }^{1}$ Kevin Gurr, MD, ${ }^{1}$ Stewart I. Bailey, MD, ${ }^{1}$ \\ Fawaz Siddiqi, MD, ${ }^{1}$ and Christopher S. Bailey, MD, MSc ${ }^{1}$
}

\begin{abstract}
${ }^{1}$ Division of Orthopaedics, Department of Surgery, Schulich School of Medicine and Dentistry, The University of Western Ontario, Lawson Health Research Institute, and London Health Sciences Centre, London, Ontario; and ${ }^{2}$ Vancouver Hospital and Health Sciences, University of British Columbia, Vancouver, British Columbia, Canada
\end{abstract}

OBJECTIVE A multicenter, prospective, randomized equivalence trial comparing a thoracolumbosacral orthosis (TLSO) to no orthosis (NO) in the treatment of acute AO Type A3 thoracolumbar burst fractures was recently conducted and demonstrated that the two treatments following an otherwise similar management protocol are equivalent at 3 months postinjury. The purpose of the present study was to determine whether there was a difference in long-term clinical and radiographic outcomes between the patients treated with and those treated without a TLSO. Here, the authors present the 5 - to 10 -year outcomes (mean follow-up $7.9 \pm 1.1$ years) of the patients at a single site from the original multicenter trial.

METHODS Between July 2002 and January 2009, a total of 96 subjects were enrolled in the primary trial and randomized to two groups: TLSO or NO. Subjects were enrolled if they had an AO Type A3 burst fracture between T-10 and L-3 within the previous 72 hours, kyphotic deformity $<35^{\circ}$, no neurological deficit, and an age of $16-60$ years old. The present study represents a subset of those patients: 16 in the TLSO group and 20 in the NO group. The primary outcome measure was the Roland Morris Disability Questionnaire (RMDQ) score at the last 5- to 10-year follow-up. Secondary outcome measures included kyphosis, satisfaction, the Numeric Rating Scale for back pain, and the 12-Item Short-Form Health Survey (SF-12) Mental and Physical Component Summary (MCS and PCS) scores. In the original study, outcome measures were administered at admission and 2 and 6 weeks, 3 and 6 months, and 1 and 2 years after injury; in the present extended follow-up study, the outcome measures were administered 5-10 years postinjury. Treatment comparison between patients in the TLSO group and those in the NO group was performed at the latest available follow-up, and the time-weighted average treatment effect was determined using a mixed-effects model of longitudinal regression for repeated measures averaged over all time periods. Missing data were assumed to be missing at random and were replaced with a set of plausible values derived using a multiple imputation procedure.

RESULTS The RMDQ score at 5-10 years postinjury was $3.6 \pm 0.9$ (mean \pm SE) for the TLSO group and $4.8 \pm 1.5$ for the NO group ( $p=0.486,95 \% \mathrm{Cl}-2.3$ to 4.8 ). Average kyphosis was $18.3^{\circ} \pm 2.2^{\circ}$ for the TLSO group and $18.6^{\circ} \pm 3.8^{\circ}$ for the NO group ( $p=0.934,95 \% \mathrm{Cl}-7.8$ to 8.5 ). No differences were found between the NO and TLSO groups with time-weighted average treatment effects for RMDQ $1.9(95 \% \mathrm{Cl}-1.5$ to 5.2$)$, for PCS $-2.5(95 \% \mathrm{Cl}-7.9$ to 3.0$)$, for $\mathrm{MCS}$ $-1.2(95 \% \mathrm{Cl}-6.7$ to 4.2$)$ and for average pain $0.9(95 \% \mathrm{Cl}-0.5$ to 2.2$)$.

CONCLUSIONS Compared with patients treated with a TLSO, patients treated using early mobilization without orthosis maintain similar pain relief and improvement in function for $5-10$ years.

https://thejns.org/doi/abs/10.3171/2016.11.SPINE161031

KEY WORDS thoracolumbar; burst fracture; thoracolumbosacral orthosis; spine; trauma

ABBREVIATIONS MCS = Mental Component Summary; NO = no orthosis; NRS = Numeric Rating Scale; PCS = Physical Component Summary; RMDQ = Roland Morris Disability Questionnaire; TLSO = thoracolumbosacral orthosis.

SUBMITTED August 25, 2016. ACCEPTED November 21, 2016.

INCLUDE WHEN CITING Published online April 14, 2017; DOI: 10.3171/2016.11.SPINE161031.

* Drs. Urquhart and Alrehaili contributed equally to this work. 
$\mathrm{T}$ HORACOLUMBAR burst fractures without neurological deficit result in a relatively stable injury because both the anterior and the middle columns of the spine fail under a compression load without associated distraction, shear, rotation, or translational injury. 7,14 Treatment of the neurologically intact patient is controversial but is generally nonsurgical given that surgery has not demonstrated superiority over nonoperative treatment with respect to pain and functional outcomes or cost-effectiveness. ${ }^{1,2,9,21,25}$ Nonoperative treatment has evolved from long-term bed rest in the hospital to early mobilization in an adjustable thoracolumbosacral orthosis (TLSO). ${ }^{5,6,11 \text {, }}$ $13,15,16,19,20,22,24$ The inherent stability of the fracture and the limitations associated with the use of a brace, including accessibility, cost, resource utilization, and patient deconditioning, lead to the question of whether a brace is even necessary. ${ }^{4}$

In a recent multicenter prospective randomized trial, we compared the treatment of AO Type A3 burst fractures between T10 and L-3 with kyphotic deformity less than $35^{\circ}$ either with or without a TLSO. ${ }^{3,4}$ The trial showed that treatment with a TLSO is equivalent to treatment without an orthosis out to 24 months postinjury according to our primary outcome measure, the Roland Morris Disability Questionnaire (RMDQ), as well as all other secondary outcome measures. Thus, in select patients with a thoracolumbar burst fracture, progressing to early ambulation with education and directed rehabilitation is both safe and equally effective with or without a brace.

Concerns about treatment without an orthosis include loss of spinal alignment, increased risk of neurological deterioration, and worse pain and functional outcome; no differences in these factors between patients with TLSO and those without were observed over the 2-year followup period in our study. No studies have reported on the long-term outcomes of early ambulation without orthosis. Therefore, to determine whether the 2-year outcomes would be sustained over time, we conducted a 5- to 10year follow-up of patients at a single site from the original multicenter trial. We hypothesized that, on longer-term follow-up, patients treated without an orthosis would be functionally and radiographically similar to those treated with a conventional TLSO.

\section{Methods \\ Study Design}

The study design of the primary trial, which was registered with the ClinicalTrials.gov database (NCT01741168), has been described in detail elsewhere. ${ }^{3,4}$ Briefly, 110 patients were screened at three trial centers between July 2002 and January 2009. Patients were eligible for inclusion if they had an isolated AO Type A3 burst fracture between T-10 and L-3 within the previous 3 days, kyphotic deformity $<35^{\circ}$, no neurological deficit, and an age of 16-60 years old. The AO Type A3 fractures are considered relatively stable burst fractures that exclude the mechanism of flexion-distraction or rotation-shear (AO Type B and C fractures, respectively). ${ }^{14,18}$ As such, the posterior ligamentous complex is considered to be intact. Participants were randomly assigned by 1:1 allocation to the TLSO or no orthosis (NO) treatment group based on a concealed, computer-generated, site-specific randomization list. Subjects were stratified based on workers' compensation status and severity of kyphotic deformity $\left(<20^{\circ} \mathrm{vs} \geq 21^{\circ}\right)$. Patients in the TLSO group were maintained on strict bed rest until fitted with a TLSO (Aspen Medical Products). Afterward, they were mobilized in the brace by a physiotherapist. All patients were instructed to wear the brace at all times, except when lying down, for a total of 10 weeks and to begin weaning from the brace at 8 weeks. Patients randomized to the NO group were mobilized immediately as tolerated by a physiotherapist with restrictions to limit bending and rotating through their trunk. They were encouraged to return to normal activities after 8 weeks.

\section{Extended Follow-Up}

The 36 subjects enrolled at one of the three study sites were contacted 5-10 years after their injury and were asked to participate in a posttrial follow-up study. Study approval was obtained from our institutional research ethics board.

Our primary outcome measure remained the RMDQ, which was administered at the last follow-up a minimum of 5 years postinjury. Secondary outcome measures included the 12-Item Short-Form Health Survey (SF-12) Mental Component Summary (MCS) and Physical Component Summary (PCS) scores (SF-36 in the original study), satisfaction, Numeric Rating Scale (NRS) for back pain, and posttraumatic kyphosis. In the original study, the outcome measures were administered at admission and 2 and 6 weeks, 3 and 6 months, and 1 and 2 years after injury; in the present extended follow-up study, outcome measures were administered 5-10 years postinjury.

The RMDQ evaluates 24 items to derive a score that varies between 0 (no disability) and 24 (severe disability). ${ }^{17}$ The SF-36 and SF-12 scores range from 0 to 100, with higher scores indicating less severe symptoms..$^{10}$ The SF36 was used during the initial 2-year follow-up period, and the SF-12 was administered at the 5- to 10-year follow-up. The SF-12 and SF-36 summary scores are highly correlated and directly comparable. ${ }^{12,23}$ The NRS for back pain ranged from 0 to 10 , with lower scores indicating less severe symptoms. Satisfaction with treatment was assessed on a 7-point scale, with 7 indicating complete satisfaction. Kyphosis was measured based on the Cobb technique as the angle measured between the perpendicular to the superior and inferior endplate of the vertebral body above and below the fractured level, respectively.

\section{Statistical Analysis}

Data analysis was performed using PAWS Statistics version 23 (SPSS Inc.). Chi-square tests and unpaired ttests were used to compare the present study participants to the cohort of patients from the primary trial not included in the present study (36 vs 60 patients), and to compare the present study participants with missing data at the final follow-up to those who had complete data (13 vs 23 patients). Missing data were assumed to be missing at random and were replaced with a set of plausible values derived using a multiple imputation procedure with 10 it- 
erations, taking all time points into consideration. Treatment comparison between patients in the TLSO group and those in the NO group was performed at the latest available follow-up (5-10 years) using a Student t-test.

For the secondary analysis, the time-weighted average treatment effect was determined with complete cases analysis (that is, using the original data without imputed data) using a mixed-effects model of longitudinal regression for repeated measures averaged over all time periods (admission and 2 and 6 weeks, 3 and 6 months, and 1,2, and 5-10 years postinjury). The main effects of time and treatment as well as time and treatment interactions were assessed, accounting for the correlation among the outcome scores in the same patient. Treatment (NO vs TLSO) and time (continuous) were included as fixed variables, and subject was included as a random variable. A comparative analysis was performed using the imputed full data set. A p value $<0.05$ was considered statistically significant. No adjustments were made for multiple comparisons.

\section{Results}

All 36 patients from one of the sites participating in the original trial were enrolled in the long-term follow-up. Sixteen patients had been randomized to the TLSO group and 20 to the NO group. For the long-term follow-up, 23 patients had data, 8 were lost to follow-up, 2 declined to participate, 2 were out of the country, and 1 was deceased. There were no differences in baseline characteristics between the subjects who returned for a long-term follow-up (23 patients) and those who did not (13 patients). The average follow-up among those participating in the long-term study was $7.9 \pm 1.1$ (range 5.9-9.5 years). The proportion of enrollees who supplied data at each follow-up visit was $100 \%$ at admission and 2 weeks postinjury, $97 \%$ at 6 weeks and 3 months, $92 \%$ at 6 and 12 months, $86 \%$ at 2 years, and $64 \%$ at 5-10 years. Of the 23 patients who completed the long-term follow-up, 25\% (3 of 12) in the NO group and $18.2 \%$ ( 2 of 11$)$ in the TLSO group reported a change in their work status to modified, short-term disability, or long-term disability $(\mathrm{p}=0.692)$. Regular medication for back pain was used by $8.3 \%$ (1 of 12) of patients in the NO group and $18.2 \%$ (2 of 11) of patients in the TLSO group (p $=0.774$ ). Patients participating in the long-term follow-up were more likely to smoke, had more pain at baseline, and were less likely to receive compensation than the patient cohorts from the other primary trial sites not included in the present study. However, comparisons of patient-rated outcome measures at the 12-month follow-up visit were not different between the two groups (Table 1).

At the long-term follow-up, the RMDQ score was 3.6 \pm 0.9 (mean \pm standard error) for the TLSO group and $4.8 \pm 1.5$ for the NO group ( $\mathrm{p}=0.486,95 \% \mathrm{CI}-2.3$ to 4.8). No differences in pain, PCS, and MCS scores were found between the TLSO and NO groups (Table 2). At the long-term follow-up, satisfaction in the TLSO group was comparable to that in the NO group ( $p=0.113)$. Average kyphosis was similar between the two groups at $5-10$ years postinjury $\left(18.3^{\circ} \pm 2.2^{\circ}\right.$ for TLSO group and $18.6^{\circ} \pm$ $3.8^{\circ}$ for $\mathrm{NO}$ group, $\mathrm{p}=0.934,95 \% \mathrm{CI}-7.8$ to 8.5 ).

The overall RMDQ was not significantly different be-
TABLE 1. Comparison of baseline characteristics and outcomes for patients enrolled in the primary trial and included in the current study versus those not included in the current study

\begin{tabular}{|c|c|c|c|}
\hline Parameter & $\begin{array}{l}\text { Current } \\
\text { Study Site }\end{array}$ & $\begin{array}{l}\text { Sites Not Included } \\
\text { in Current Study }\end{array}$ & $\begin{array}{c}\mathrm{p} \\
\text { Value }\end{array}$ \\
\hline No. of patients & 36 & 60 & \\
\hline Mean age in yrs & $40.8 \pm 16.3$ & $39.7 \pm 14.2$ & 0.740 \\
\hline No. of women (\%) & $14(38.9)$ & $15(25.0)$ & 0.151 \\
\hline $\begin{array}{l}\text { Mean admission kyphosis } \\
\text { in degrees }\end{array}$ & $12.7 \pm 5.1$ & $14.9 \pm 7.9$ & 0.213 \\
\hline No. w/ kyphosis $\geq 20^{\circ}(\%)$ & $6(16.7)$ & $4(6.7)$ & 0.120 \\
\hline No. of smokers (\%) & $18(50.0)$ & $13(21.7)$ & 0.004 \\
\hline \multicolumn{4}{|l|}{ Level of fracture (no. [\%]) } \\
\hline $\mathrm{T}-11$ & $3(8.3)$ & $1(1.7)$ & 0.080 \\
\hline $\mathrm{T}-12$ & $10(27.8)$ & $8(13.3)$ & \\
\hline L-1 & $18(50.0)$ & $32(53.3)$ & \\
\hline $\mathrm{L}-2$ & $4(11.1)$ & $11(18.3)$ & \\
\hline L-3 & $1(2.8)$ & $8(13.3)$ & \\
\hline Compensation* (no. [\%]) & $5(13.9)$ & $24(40.0)$ & 0.005 \\
\hline RMDQ at enrollment & $18.6 \pm 5.3$ & $17.3 \pm 5.3$ & 0.243 \\
\hline SF-36 PCS at enrollment & $28.0 \pm 9.1$ & $29.7 \pm 10.9$ & 0.431 \\
\hline SF-36 MCS at enrollment & $49.5 \pm 11.4$ & $51.2 \pm 12.8$ & 0.523 \\
\hline Average pain at enrollment & $6.5 \pm 2.7$ & $5.2 \pm 2.2$ & 0.018 \\
\hline Satisfaction at enrollment & $6.3 \pm 1.7$ & $6.6 \pm 1.4$ & 0.470 \\
\hline RMDQ at $12-m o ~ F U$ & $5.0 \pm 6.2$ & $3.7 \pm 4.6$ & 0.276 \\
\hline SF-36 PCS at 12-mo FU & $44.5 \pm 11.8$ & $45.8 \pm 9.8$ & 0.603 \\
\hline SF-36 MCS at 12-mo FU & $53.0 \pm 9.1$ & $52.5 \pm 8.5$ & 0.806 \\
\hline Average pain at $12-\mathrm{mo} \mathrm{FU}$ & $2.3 \pm 2.3$ & $1.6 \pm 1.5$ & 0.123 \\
\hline Satisfaction at 12-mo FU & $6.3 \pm 1.7$ & $6.5 \pm 1.3$ & 0.609 \\
\hline
\end{tabular}

tween TLSO and NO from admission to 5-10 years postinjury ( $\mathrm{p}=0.161$; Table 3 and Fig. 1A). Likewise, no difference was found between the time-weighted average treatment effects for all other secondary outcomes (Fig. 1B-E). Repeat analysis using multiple imputation for missing data showed no significant differences between the TLSO and NO groups. Treatment effect estimates for the multiple imputation analysis ranged from 2.0 to 2.6 for RMDQ, -3.8 to -1.4 for PCS, -2.7 to -0.3 for MCS, 0.9 to 0.7 for pain, and -1.1 to 0.3 for satisfaction. In both groups, RMDQ, PCS, MCS, and pain improved with time from admission for most time points until 6 months post injury (Fig. 1; p < 0.01 at all time points up to 6 months). Overall satisfaction with treatment did not change with time $(\mathrm{p}=0.930)$.

\section{Discussion}

The longer-term follow-up results at one center from an original multicenter trial showed that, as compared with patients treated with a TLSO, those patients treated using early mobilization without orthosis maintain similar pain relief and functional improvement through 5-10 years after sustaining a stable AO Type A3 thoracolumbar burst 
TABLE 2. Comparison of mean clinical and radiographic outcomes at the final follow-up at 5-10 years postinjury

\begin{tabular}{|c|c|c|c|c|c|}
\hline Parameter & NO Group & TLSO Group & Treatment Effect & $95 \% \mathrm{Cl}$ & $\mathrm{p}$ Value \\
\hline RMDQ score* & $4.8 \pm 1.5$ & $3.6 \pm 0.9$ & 1.3 & -2.3 to 4.8 & 0.486 \\
\hline PCS score $\dagger$ & $45.0 \pm 3.8$ & $46.5 \pm 3.2$ & -1.5 & -11.7 to 8.8 & 0.778 \\
\hline MCS score $†$ & $55.2 \pm 2.4$ & $55.8 \pm 2.1$ & -0.6 & -7.8 to 6.7 & 0.868 \\
\hline Average pain score* & $2.9 \pm 0.7$ & $2.1 \pm 0.6$ & 0.9 & -1.0 to 2.8 & 0.378 \\
\hline Satisfaction score & $5.6 \pm 0.5$ & $6.5 \pm 0.4$ & -0.9 & -2.0 to 0.2 & 0.113 \\
\hline Kyphosis in degrees & $18.6 \pm 3.8$ & $18.3 \pm 2.2$ & 0.4 & -7.8 to 8.5 & 0.934 \\
\hline
\end{tabular}

fracture without neurological injury. Although many studies have shown that nonsurgical treatments such as body casting or bracing are safe and effective, $, 511,19,20$ the present study is the first to report on the long-term outcomes in treating these fractures without any external orthosis.

Our study revealed that patients treated with or without a TLSO can expect a significant improvement of symptoms in terms of RMDQ and pain over the first 6 months to 1 year, with little or no further change between 2 and 10 years after injury. The favorable clinical outcomes reported here are in agreement with those in previous studies of conservative treatment. ${ }^{9,11,25}$ In a randomized clinical trial by Wood et al., ${ }^{25}$ patients treated nonoperatively with a cast or brace were compared with those treated surgically at 4 and 20 years' follow-up. At 4 years after injury, the nonsurgically treated patients had residual disability (median RMDQ Score 2, range 0-24) and pain (median visual analog scale score $1.5 \mathrm{~cm}$, range $0-9 \mathrm{~cm}$ ) similar to that in our patients but a slight improvement in function according to the RMDQ score between the 4- and 20-year time points (median RMDQ Score 1, range 0-9). Furthermore, in the present study, at 5-10 years after injury, there was no deterioration in ability or increased pain in patients treated without orthosis compared to those treated with TLSO.

Consistent with the clinical outcome, patients treated with or without orthosis returned to work and required

TABLE 3. Overall means and treatment effects of secondary outcomes averaged across all follow-up time points during the 5-10 years of follow-up

\begin{tabular}{lrrr}
\hline \multicolumn{1}{c}{ Parameter } & \multicolumn{1}{c}{$\begin{array}{c}\text { NO } \\
\text { Group }\end{array}$} & \multicolumn{1}{c}{$\begin{array}{c}\text { TLSO } \\
\text { Group }\end{array}$} & \multicolumn{1}{c}{$\begin{array}{c}\text { Treatment Effect } \\
(95 \% \mathrm{Cl})\end{array}$} \\
\hline RMDQ score* $^{*}$ & $10.0 \pm 1.1$ & $8.1 \pm 1.2$ & $1.9(-1.5$ to 5.2$)$ \\
\hline PCS score $\dagger$ & $37.1 \pm 1.8$ & $39.6 \pm 1.9$ & $-2.5(-7.9$ to 3.0$)$ \\
\hline MCS score $\dagger$ & $51.3 \pm 1.7$ & $52.5 \pm 2.0$ & $-1.2(-6.7$ to 4.2$)$ \\
\hline Average pain score* & $3.6 \pm 0.4$ & $2.8 \pm 0.5$ & $0.9(-0.5$ to 2.2$)$ \\
\hline Satisfaction score & $6.2 \pm 0.2$ & $6.7 \pm 0.2$ & $-0.4(-1.1$ to 0.3$)$ \\
\hline Kyphosis in degrees & $19.1 \pm 1.9$ & $17.8 \pm 2.1$ & $2.0(-3.8$ to 7.8$)$ \\
\hline
\end{tabular}

Values expressed as the mean \pm standard error, unless indicated otherwise. Overall mean, standard error, treatment effect, and $95 \%$ confidence interval are derived using mixed-effects model for repeated measures.

* Lower score indicates less severe symptoms.

$\dagger$ Mean summary scores are based on normative data and have a mean \pm standard deviation of $50 \pm 10$. minimal medications for back pain. Furthermore, regardless of the treatment modality, patients continued to be satisfied with their treatment at 5-10 years after injury.

Radiographic analysis revealed, regardless of treatment modality, an average progression of approximately $7^{\circ}$ in the kyphosis angle from admission (NO $12^{\circ}$ vs TLSO $13^{\circ}$ ) to 2 weeks after injury (NO $17^{\circ}$ vs $\operatorname{TLSO} 18^{\circ}$ ) and little or no progression during the 5-10 years' follow-up (NO $19^{\circ}$ vs TLSO $18^{\circ}$ ). Likewise, in a cohort study of 60 patients treated conservatively (brace), Jaffray et al. ${ }^{11}$ reported an average increase in kyphotic deformity from $17^{\circ}$ (range $5^{\circ}-29^{\circ}$ ) to $20^{\circ}$ (range $1^{\circ}-28^{\circ}$ ) from admission to 3 months postinjury. The average kyphosis documented by Wood et al..$^{25}$ at 20 years after injury was $19^{\circ}$ for conservatively treated patients. Our results are consistent with those of previous studies showing the natural history of thoracolumbar burst fractures more than 10 years after injury and confirm no increased loss of spinal alignment in patients treated with or without an external orthosis.

We acknowledge that this study has some limitations. First, our analysis included participants from one site of a larger trial (within-subgroup effect). Therefore, the number of patients was not prespecified, so ensuring adequate statistical power was not possible. However, to minimize the selection bias and baseline incomparability inherent in a subgroup analysis, end points were predefined and treatment assignment was based on a site-specific randomization list. ${ }^{8}$ Moreover, patients in the present study were more likely to smoke, had more pain at baseline, and were less likely to receive compensation than the cohort of patients from the primary trial that was not included in the present study. These potentially prognostic factors for this patient population were probably not influential as no difference between treatment groups emerged in the long-term follow-up. Additionally, post hoc comparison with the cohort from the other primary trial sites revealed no differences in 12-month outcome measures. Nonetheless, these behavioral traits should be considered when applying these findings to the general population. Second, we were unable to obtain all outcomes for all participants because of loss to follow-up. However, the longitudinal mixed-effects models used in the main analysis relied on the population average of all available data while accounting for missing values at random. In addition, analysis using a multiple imputation procedure yielded consistent results. Third, satisfaction was much higher than reported 

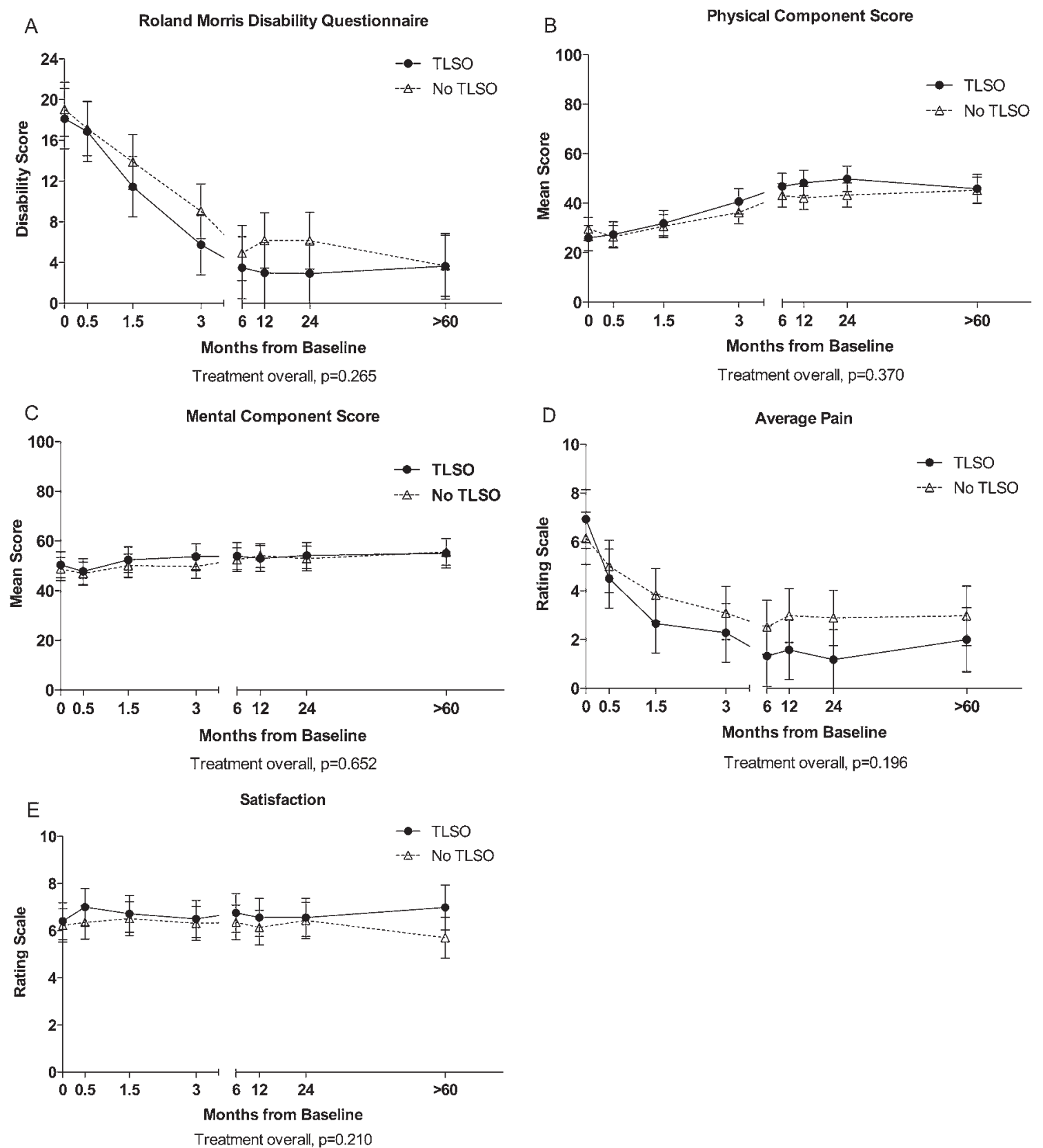

Treatment overall, $\mathrm{p}=0.196$

FIG. 1. Outcomes at each follow-up visit through the long-term follow-up of 5-10 years: RMDQ (A), PCS (B), MCS (C), average pain (D), and satisfaction with symptoms (E). Error bars indicate the $95 \%$ confidence interval.

previously, ${ }^{6}$ probably because there was a ceiling effect, with the majority of patients reporting 6 out of a maximum score of 7 at baseline.

\section{Conclusions}

In summary, the results of this study reassure health care professionals that treating a thoracolumbar burst fracture patient with early ambulation is both safe and effective with or without a TLSO through 5-10 years postinjury.

\section{References}

1. Aleem IS, Nassr A: Cochrane in CORR ${ }^{\circledR}$ : Surgical versus non-surgical treatment for thoracolumbar burst fractures without neurological deficit. Clin Orthop Relat Res 474:619-624, 2016

2. Aras EL, Bunger C, Hansen ES, Søgaard R: Cost-effectiveness of surgical versus conservative treatment for throacolumbar burst fractures. Spine (Phila Pa 1976) 41:337-343, 2016

3. Bailey CS, Dvorak MF, Thomas KC, Boyd MC, Paquett S, Kwon BK, et al: Comparison of thoracolumbosacral orthosis and no orthosis for the treatment of thoracolumbar burst fractures: interim analysis of a multicenter randomized clinical equivalence trial. J Neurosurg Spine 11:295-303, 2009

4. Bailey CS, Urquhart JC, Dvorak MF, Nadeau M, Boyd MC, Thomas KC, et al: Orthosis versus no orthosis for the treatment of thoracolumbar burst fractures without neurologic 
injury: a multicenter prospective randomized equivalence trial. Spine J 14:2557-2564, 2014

5. Cantor JB, Lebwohl NH, Garvey T, Eismont FJ: Nonoperative management of stable thoracolumbar burst fractures with early ambulation and bracing. Spine (Phila Pa 1976) 18:971-976, 1993

6. Chow GH, Nelson BJ, Gebhard JS, Brugman JL, Brown CW, Donaldson DH: Functional outcome of thoracolumbar burst fractures managed with hyperextension casting or bracing and early mobilization. Spine (Phila Pa 1976) 21:2170-2175, 1996

7. Denis F: The three column spine and its significance in the classification of acute thoracolumbar spinal injuries. Spine (Phila Pa 1976) 8:817-831, 1983

8. Dijkman B, Kooistra B, Bhandari M: How to work with a subgroup analysis. Can J Surg 52:515-522, 2009

9. Gnanenthiran SR, Adie S, Harris IA: Nonoperative versus operative treatment for thoracolumbar burst fractures without neurologic deficit: a meta-analysis. Clin Orthop Relat Res 470:567-577, 2012

10. Grevitt M, Khazim R, Webb J, Mulholland R, Shepperd J: The Short Form-36 Health Survey questionnaire in spine surgery. J Bone Joint Surg Br 79:48-52, 1997

11. Jaffray DC, Eisenstein SM, Balain B, Trivedi JM, Newton Ede M: Early mobilisation of thoracolumbar burst fractures without neurology: a natural history observation. Bone Joint J 98-B:97-101, 2016

12. Jenkinson C, Layte R, Jenkinson D, Lawrence K, Petersen S, Paice C, et al: A shorter form health survey: can the SF-12 replicate results from the SF-36 in longitudinal studies? J Public Health Med 19:179-186, 1997

13. Krompinger WJ, Fredrickson BE, Mino DE, Yuan HA: Conservative treatment of fractures of the thoracic and lumbar spine. Orthop Clin North Am 17:161-170, 1986

14. Magerl F, Aebi M: A comprehensive classification of thoracic and lumbar injuries, in Aebi M, Thalgott JS, Webb JK (eds): AO ASIF Principles in Spine Surgery. Berlin: Springer, 1998, pp 20-41

15. Moller A, Hasserius R, Redlund-Johnell I, Ohlin A, Karlsson MK: Nonoperatively treated burst fractures of the thoracic and lumbar spine in adults: a 23 - to 41-year follow-up. Spine J 7:701-707, 2007

16. Mumford J, Weinstein JN, Spratt KF, Goel VK: Thoracolumbar burst fractures. The clinical efficacy and outcome of nonoperative management. Spine (Phila Pa 1976) 18:955-970, 1993

17. Roland M, Fairbank J: The Roland-Morris Disability Questionnaire and the Oswestry Disability Questionnaire. Spine (Phila Pa 1976) 25:3115-3124, 2000

18. Schroeder GD, Kepler CK, Koerner JD, Chapman JR, Bellabarba $\mathrm{C}$, Oner FC, et al: Is there a regional difference in morphology interpretation of A3 and A4 fractures among different cultures? J Neurosurg Spine 24:1-8, 2015
19. Shamji MF, Roffey DM, Young DK, Reindl R, Wai EK: A pilot evaluation of the role of bracing in stable thoracolumbar burst fractures without neurological deficit. J Spinal Disord Tech 27:370-375, 2014

20. Shen WJ, Shen YS: Nonsurgical treatment of three-column thoracolumbar junction burst fractures without neurologic deficit. Spine (Phila Pa 1976) 24:412-415, 1999

21. Thomas KC, Bailey CS, Dvorak MF, Kwon B, Fisher C: Comparison of operative and nonoperative treatment for thoracolumbar burst fractures in patients without neurological deficit: a systematic review. J Neurosurg Spine 4:351-358, 2006

22. Tropiano P, Huang RC, Louis CA, Poitout DG, Louis RP: Functional and radiographic outcome of thoracolumbar and lumbar burst fractures managed by closed orthopaedic reduction and casting. Spine (Phila Pa 1976) 28:2459-2465, 2003

23. Ware J Jr, Kosinski M, Keller SD: A 12-Item Short-Form Health Survey: construction of scales and preliminary tests of reliability and validity. Med Care 34:220-233, 1996

24. Weinstein JN, Collalto P, Lehmann TR: Thoracolumbar "burst" fractures treated conservatively: a long-term followup. Spine (Phila Pa 1976) 13:33-38, 1988

25. Wood KB, Buttermann GR, Phukan R, Harrod CC, Mehbod A, Shannon B, et al: Operative compared with nonoperative treatment of a thoracolumbar burst fracture without neurological deficit: a prospective randomized study with followup at sixteen to twenty-two years. J Bone Joint Surg Am 97:3-9, 2015

\section{Disclosures}

Dr. Fisher has been a consultant for Medtronic and NuVasive; has received royalties from Medtronic; and has received fellowship program support from Medtronic and AOSpine.

\section{Author Contributions}

Conception and design: C Bailey. Acquisition of data: C Bailey, Alrehaili, Rasoulinejad, Gurr, S Bailey. Analysis and interpretation of data: C Bailey, Urquhart. Drafting the article: C Bailey, Urquhart. Critically revising the article: C Bailey, Fisher, Rasoulinejad. Reviewed submitted version of manuscript: all authors. Approved the final version of the manuscript on behalf of all authors: C Bailey. Statistical analysis: Urquhart. Administrative/ technical/material support: Urquhart, Alrehaili, Fleming. Study supervision: C Bailey.

\section{Correspondence}

Christopher Bailey, London Health Sciences Centre, E4-120, 800 Commissioners Rd. East, London, ON N6A 4G5, Canada. email: chris.bailey@lhsc.on.ca. 